\title{
Thin endometrial thickness is a risk factor for singleton low birth weight from single blastocyst transfer: A retrospective cohort study
}

\author{
Mingze $\mathrm{Du}^{1}$, Junwei Zhang ${ }^{2}$, Manman $\mathrm{Liu}^{3}$, and Xingling Wang ${ }^{3}$ \\ ${ }^{1}$ Third affiliated hospital of Zhengzhou University \\ ${ }^{2}$ The Third Affiliated Hospital of Zhengzhou University \\ ${ }^{3}$ Affiliation not available
}

May 26, 2021

\begin{abstract}
Objective To explore whether thin endometrial thickness (EMT) is associated with singleton low birth weight (LBW) from single fresh blastocyst transfer. Design Retrospective cohort study. Setting Reproductive center. Population All women were [?]40 years old and underwent single fresh blastocyst transfer and singleton live birth. Interventions None. Methods Multivariable logistic regression was used to evaluate the association between thin EMT and singleton LBW. Main outcome measures LBW was the primary concern of this study. Results In total, 2847 women met the study inclusion criteria. The neonatal birthweight in the EMT [?]7.5 mm group was significantly lower than that in the EMT 7.6 $\sim 12.0 \mathrm{~mm}$ and EMT $>12.0 \mathrm{~mm}$ group $(\mathrm{P}<0.001)$. The rate of LBW in the EMT [?]7.5 mm group was $24.9 \%$, which was significantly higher than the $4.0 \%$ in the EMT $7.6 \sim 12.0$ $\mathrm{mm}$ group and the $5.3 \%$ in the EMT $>12.0 \mathrm{~mm}$ group $(\mathrm{P}<0.001)$. The total neonatal malformation rate was similar between the groups $(1.1 \%, 0.8 \%$ and $1.5 \%, \mathrm{P}=0.21)$. After multiple logistic regression analysis, EMT[?]7.5 mm was an independent risk factor for LBW (adjusted odds ratio [AOR]: 4.39, 95\% CI: 1.85 10.46, P<0.001). Conclusions Thin EMT ([?]7.5 mm) on the hCG trigger day is an independent risk factor for singleton LBW from single fresh blastocyst transfer. The neonatal birthweight in the EMT [?]7.5 mm group was significantly lower than that in the EMT 7.6 $12.0 \mathrm{~mm}$ and EMT $>12.0 \mathrm{~mm}$ groups. The total neonatal malformation rate was comparable between the groups.
\end{abstract}

Title page

Title

Thin endometrial thickness is a risk factor for singleton low birth weight from single blastocyst transfer: A retrospective cohort study

\section{Running title}

Endometrium thickness is a risk factor for LBW

\section{Authors}

Mingze $\mathrm{Du}^{1+}$, Junwei Zhang ${ }^{1+}$, Manman $\mathrm{Liu}^{1}$, Xingling Wang ${ }^{1 *}$

${ }^{1}$ The Reproduction Center, The Third Affiliated Hospital of Zhengzhou University, 7 Kangfuqian Road, Zhengzhou, 450052, Henan, People's Republic of China

${ }^{*}$ Corresponding author: Xingling Wang

Correspondence address: The Reproduction Center, The Third Affiliated Hospital of Zhengzhou University, 7 Kangfuqian Road, Zhengzhou, 450052, Henan, People's Republic of China 
E-mail: wangxl1616@126.com

Phone numbers: $86+15617679237$

Present/permanent address: The Reproduction Center, The Third Affiliated Hospital of Zhengzhou University, 7 Kangfuqian Road, Zhengzhou, 450052, Henan, People's Republic of China

${ }^{+}$These authors have contributed equally to this work and share first authorship

\section{Abstract}

Objective To explore whether thin endometrial thickness (EMT) is associated with singleton low birth weight (LBW) from single fresh blastocyst transfer.

Design Retrospective cohort study.

Setting Reproductive center.

Population All women were [?]40 years old and underwent single fresh blastocyst transfer and singleton live birth.

Interventions None.

Methods Multivariable logistic regression was used to evaluate the association between thin EMT and singleton LBW.

Main outcome measures LBW was the primary concern of this study.

Results In total, 2847 women met the study inclusion criteria. The neonatal birthweight in the EMT [?]7.5 $\mathrm{mm}$ group was significantly lower than that in the EMT 7.6 $12.0 \mathrm{~mm}$ and EMT $>12.0 \mathrm{~mm}$ group $(\mathrm{P}<0.001)$. The rate of LBW in the EMT [?]7.5 mm group was $24.9 \%$, which was significantly higher than the $4.0 \%$ in the EMT 7.6 $12.0 \mathrm{~mm}$ group and the $5.3 \%$ in the EMT $>12.0 \mathrm{~mm}$ group $(\mathrm{P}<0.001)$. The total neonatal malformation rate was similar between the groups $(1.1 \%, 0.8 \%$ and $1.5 \%, \mathrm{P}=0.21)$. After multiple logistic regression analysis, EMT[?]7.5 mm was an independent risk factor for LBW (adjusted odds ratio [AOR]: 4.39, 95\% CI: 1.85 10.46, $\mathrm{P}<0.001)$.

Conclusions Thin EMT ([?]7.5 mm) on the hCG trigger day is an independent risk factor for singleton LBW from single fresh blastocyst transfer. The neonatal birthweight in the EMT [?]7.5 mm group was significantly lower than that in the EMT $7.6^{\sim} 12.0 \mathrm{~mm}$ and EMT $>12.0 \mathrm{~mm}$ groups. The total neonatal malformation rate was comparable between the groups.

Keywords low birth weight, endometrial thickness, blastocyst, birthweight

Tweetable abstract Thin endometrium thickness is an independent risk factor for singleton LBW from single fresh blastocyst transfer.

Introduction

Infertility affects approximately $10 \%$ of reproductive-aged couples worldwide ${ }^{1}$. In vitro fertilization (IVF) is widely used and has become the most effective treatment for infertility caused by tubal factors or other factors as well as unexplained infertility ${ }^{2}$. The safety of IVF offspring has always been our focus. However, adverse pregnancy and perinatal outcomes, such as low birth weight (LBW), preterm birth, pregnancyinduced hypertension and gestational diabetes, are increased, even for singleton births ${ }^{3-5}$. However, the exact biological mechanism leading to adverse perinatal outcomes is unclear. Some studies have shown that infertility disease itself is the main reason for the poor perinatal outcome of assisted reproductive technology (ART) singleton offspring ${ }^{6}$. However, an increasing number of studies have shown that the process of ART, including exposure to superphysiological doses of hormones and embryo manipulation in the laboratory, may have an adverse effect on the perinatal outcome ${ }^{7-10}$. Furthermore, defective placentation, particularly in patients with thin endometrium thickness (EMT), may be an important cause of these poor perinatal outcomes $^{11,12}$. However, there is no exact definition of "thin EMT", and most studies have reported cutoff 
values of $7 \mathrm{~mm}, 7.5 \mathrm{~mm}$ or $8 \mathrm{~mm}^{13-17}$. Thin EMT is an independent factor affecting the ART pregnancy rate $^{18}$. In recent years, studies have shown that a thin EMT is not only related to the pregnancy rate but also to perinatal complications and offspring safety ${ }^{13-15}$. However, there are few related studies and limited data, and cleavage-stage embryos and blastocysts have not been studied and analyzed separately. Different stages of embryo development may have different effects on offspring safety ${ }^{19,}{ }^{20}$. Therefore, in this study, we selected patients with single fresh blastocyst transfer and analyzed the effect of thin EMT on the LBW of singleton offspring.

Materials and methods

Study design and population

A retrospective cohort study was approved by the review board of The Third Affiliated Hospital of Zhengzhou University. All patients who initiated their first in vitro fertilization (IVF)/intracytoplasmic sperm injection (ICSI) cycles at the Reproductive Center of Third Affiliated Hospital of Zhengzhou University between January 2016 and February 2020 were analyzed for potential inclusion. Inclusion criteria for women aged [?]40 years, undergoing single fresh blastocyst transfer (D5/D6) and having a singleton live birth. Cycles with oocyte donation, vanishing twins, adenomyosis, uterine malformations, endometrial polyps, preimplantation genetic testing (PGT) or incomplete records were excluded.

Clinical and laboratory protocols

All of the women were undergoing standardized GnRH agonist (GnRH-a) or flexible GnRH antagonist (GnRH-anti) protocols. The details of controlled ovarian hyperstimulation $(\mathrm{COH})$ were described in our previous study ${ }^{3}$. In brief, for the GnRH-a protocol, protracted downregulation with GnRH-a (Diphereline, lpsen, France) $3.75 \mathrm{mg}$ was performed on the second or third day of the menstrual cycle, followed by daily use of recombinant follicle-stimulating hormone ( $\mathrm{rFSH}$ ), which was based on the ovarian response. In the flexible GnRH-anti group, rFSH was initiated on the second or third day of the menstrual cycle. Injection of GnRH-anti at $0.25 \mathrm{mg} /$ day commenced once the diameter of the dominant follicle reached $14 \mathrm{~mm}$ and was continued up to the trigger day.

For both protocols, the dose of rFSH was adjusted according to the follicle response. As soon as the diameter of the dominant follicle was greater than $20 \mathrm{~mm}$ or when at least three follicles reached $18 \mathrm{~mm}$, ovulation induction was triggered with 5000 to 10000 IU hCG (Lizhu Pharmaceutical Trading, China). Oocyte retrieval was performed 36 hours later. Based on the sperm quality, conventional IVF or ICSI was performed approximately 4 to 6 hours after follicular aspiration. In our study, all women underwent single fresh blastocyst transfer on the fifth day after fertilization. Routine corpus luteum support was initially provided on the day of oocyte retrieval, namely, oral dydrogesterone (10 mg twice daily) (Abbott Co. America), and intravaginal administration of $90 \mathrm{mg}$ of a progesterone sustained-release vaginal gel (Merck Co. Germany) was given. Corpus luteum support was performed at least until 55 days after transplantation if pregnancy occurred.

EMT assessment and grouping

For EMT assessment, only three doctors with extensive experience in transvaginal ultrasound monitoring performed the EMT assessment in our reproductive center. EMT was measured in the sagittal plane. The distance between the hyperechogenic interfaces between the endometrium and the myometrium was recorded approximately 1 centimeter beneath the uterine fundus. The EMT was recorded and analyzed in millimeters. The EMT was taken from the hCG trigger day. Patients were categorized into three groups depending on their EMTs: [?]7.5 mm, 7.6 $12.0 \mathrm{~mm}$ and $>12.0 \mathrm{~mm}$. These cutoffs were selected as being close to those in previous studies ${ }^{13-15}$.

Outcome measures and definition

LBW was defined as a neonatal birth weight less than $2500 \mathrm{~g}^{21}$ and was the primary concern of this study. The secondary outcome measures were neonatal birthweight and neonatal congenital malformations, including 
trisomy 13/18/21, congenital heart disease, polydactyly/syndactyly and other disorders.

Statistical analysis

All of the data analysis in our study were obtained via review of our reproductive center's medical records.

The one-sample Kolmogorov-Smirnov test was used to check for normality of continuous variables. The Wilcoxon rank-sum test was used to assess between-group differences in continuous variables with abnormal distributions, and these variables are expressed as the median (P25, P75). Categorical variables are presented as the number of cases (n) and the percentage (\%). The means obtained from chi-square analyses were used to assess the differences between groups with Fisher's exact test when necessary. For LBW, multiple logistic regression was used to adjust for the baseline characteristics between groups. Adjusted odds ratios (AORs) with $95 \%$ confidence intervals (CIs) were calculated. All statistical management and analyses were performed using SPSS software, version 24.0. Statistical significance was set at $\mathrm{p}<0.05$.

Results

Study population

In total, 2847 women who underwent single fresh blastocyst transfer and delivered singleton births met the study inclusion criteria, including 181 women with EMT [?]7.5 mm, 1714 women with EMT 7.6 $12.0 \mathrm{~mm}$ and 952 women with EMT $>12.0 \mathrm{~mm}$.

Characteristics of the study groups

Table 1 shows the demographic and clinical characteristics among the three groups. There were no significant between-group differences in maternal age, body mass index (BMI), duration of infertility, type of infertility, infertility diagnosis, basal serum FSH level, number of antral follicle counts, fertilization method, $\mathrm{COH}$ protocols, duration of ovarian stimulation, dosage of gonadotropins, serum estradiol on the trigger day or number of oocytes retrieved.

Neonatal outcomes

The neonatal birthweight in the EMT [?]7.5 mm group was significantly lower than that in the EMT 7.6 12.0 $\mathrm{mm}$ and EMT >12.0 mm group (EMT [?]7.5 mm, 3000 (2525,3350); EMT 7.6 $12.0 \mathrm{~mm}, 3350$ (3050,3650); EMT $>12.0 \mathrm{~mm}, 3400(3100,3690), \mathrm{P}<0.001)$. The sex of the newborns was comparable between groups $(\mathrm{P}=0.06)$. The rate of LBW in the EMT [?]7.5 mm group was $24.9 \%$, which was significantly higher than the $4.0 \%$ in the EMT $7.6^{\sim} 12.0 \mathrm{~mm}$ and $5.3 \%$ in the EMT $>12.0 \mathrm{~mm}$ group $(\mathrm{P}<0.001)$. The total neonatal malformation rate was similar between the groups $(1.1 \%, 0.8 \%$ and $1.5 \%, \mathrm{P}=0.21)$.

Regarding the main outcome measures, LBW, to adjust for the influence of confounding factors, we conducted a multiple logistic regression analysis. The regression model included the following factors: maternal age (continuous variable), BMI (continuous variable), type of infertility (primary/secondary infertility), infertility diagnosis (tubal/male/others), COH protocols (GnRH-a protocol/GnRH-anti protocol), serum estradiol on the trigger day $(<4000 \mathrm{pg} / \mathrm{ml}[?] 4000 \mathrm{pg} / \mathrm{ml})$, EMT ([?]7.5/7.6 12.0/>12.0 mm), number of gestational weeks (continuous variable) and sex of the newborn (male/female). Compared with EMT 7.6 12.0 mm, EMT[?]7.5 $\mathrm{mm}$ was an independent risk factor for LBW (AOR: 4.39, 95\% CI: 1.85 10.46, $\mathrm{P}<0.001$ ). In addition, BMI (AOR: 0.91, 95\% CI: 0.84 0.99, $\mathrm{P}=0.03$ ), serum estradiol on the trigger day (AOR: 1.87, 95\% CI: 1.162 .21 , $\mathrm{P}=0.03$ ), gestational weeks (AOR: $0.37,95 \% \mathrm{CI}: 0.320 .42, \mathrm{P}<0.001$ ) and sex of the newborn (AOR: 1.99, 95\% CI: 1.19 3.33, $\mathrm{P}=0.01$ ) were risk factors for LBW. The detailed data are described in Table 3.

Discussion

Main findings

In this single-center retrospective cohort study, a thin EMT ([?]7.5 mm) on the hCG trigger day was an independent risk factor for LBW of a singleton live birth from a single fresh blastocyst transfer. The neonatal 
birthweight in the EMT [?]7.5 mm group was significantly lower than that in the EMT $7.6^{\sim} 12.0 \mathrm{~mm}$ and EMT $>12.0 \mathrm{~mm}$ groups. The total neonatal malformation rate was comparable between groups.

Strengths and limitations

The first study to explore the relationship between thin EMT and perinatal outcome was performed by Chung et al. ${ }^{22}$. This study found that EMT has a significant protective effect on perinatal outcomes, namely, LBW and preterm birth; in other words, suboptimal endometrial development is associated with adverse outcomes in pregnancies achieved through $\mathrm{IVF}^{22}$. Another early study explored the relationship between thin EMT and perinatal outcome regarding the risk of placenta previa. Rombauts et al. found that the risk of placenta previa was significantly higher in women with EMT $>12 \mathrm{~mm}$ than in women with an endometrial thickness of $<9 \mathrm{~mm}^{23}$. A large retrospective cohort study including 6181 singleton newborns found that a thin EMT was associated with a lower mean birthweight and birthweight Z-score ${ }^{15}$. Recently, Guo et al. ${ }^{13}$ reported that the risk of being small for gestational age (SGA) was increased approximately twofold in women with EMT[?]7.5 mm compared with women with EMT $>12 \mathrm{~mm}$ after fresh embryo transfer. However, to the best of our knowledge, the current study did not analyze the effect of EMT on the hCG trigger day on the neonatal birthweight from single fresh blastocyst transfer. We know that the different stages of embryo transfer, that is, the cleavage-stage embryo or the blastocyst, may have different effects on perinatal outcomes $^{19,24,25}$. However, single blastocyst transfer, compared with cleavage-stage embryo transfer, can increase the pregnancy rate, and has a lower risk of multiple births ${ }^{26}$; moreover, single blastocyst transfer is increasingly widely used in clinical practice and may become the recommended transplantation strategy. Therefore, to reduce the influence of confounding factors, it is necessary to further analyze the impact of EMT of single blastocyst transfer on the safety of the offspring. In this study, thin EMT ([?]7.5 mm) was an independent risk factor for LBW of a singleton live birth from a single fresh blastocyst transfer.

In this study, to minimize the influence of confounding factors, only single fresh blastocyst transfer was included. After analyzing and adjusting for confounding factors by EMT grouping, this study showed that thin EMT ([?]7.5 mm) is an independent risk factor for LBW and further confirmed the influence of EMT on neonatal birthweight, which is an important supplement to current clinical research. To the best of our knowledge, this is the first study to explore the effect of thin EMT on singleton LBW from single blastocyst transfer. Only single-center data were included to minimize the influence of confounding factors caused by different EMT measurements, clinical protocols, and laboratory operations. However, this investigation also has certain limitations. It was a retrospective cohort study and did not further explore the relevant biological mechanism by which thin EMT affects the incidence of LBW.

Interpretation

The specific biological mechanism of the impact of thin EMT on newborn weight and perinatal outcomes is still unclear and likely complex. Research on the main mechanism focuses on the oxygen concentration. After ovulation, the uterine spiral artery contracts, and the blood flow on the surface of the endometrium decreases, thereby reducing the oxygen concentration of the functional epithelium during embryo implantation ${ }^{27}$. In early pregnancy, hypoxic tension is one of the main conditions for normal embryo implantation and fetal development ${ }^{28}$. However, the thinning or lack of a functional layer may cause more blood vessel growth and higher oxygen concentrations in the basal endometrium of the embryo. The subsequent high oxygen tension may affect embryo implantation and placental development, thereby affecting the growth and development of the fetus ${ }^{29,30}$. Another mechanism is related to spiral arterial revascularization. In early pregnancy, spiral arterial vascular remodeling is an important factor in determining placental blood perfusion, and the lack of vascular remodeling will affect the blood supply of the placenta and eventually lead to perinatal complications, such as fetal growth restriction and pregnancy-related hypertension ${ }^{31,32}$. The uterine artery blood flow resistance of a thin endometrium is high, and there is vascular dysplasia. These changes may affect spiral artery vascular remodeling, thereby affecting the development of the placenta and causing poor perinatal outcomes ${ }^{12,15}$.

Conclusion 
In conclusion, this study showed that a thin EMT ([?]7.5 mm) was significantly correlated with neonatal birthweight and was an independent risk factor for singleton LBW from fresh single blastocyst transfer. In addition, the neonatal birth weight in the EMT [?]7.5 mm group was significantly lower than that in the EMT $7.6^{\sim} 12.0 \mathrm{~mm}$ and EMT $>12.0 \mathrm{~mm}$ groups. This finding may be related to spiral arterial vascular remodeling and placental development, and further basic research is needed to explore its related biological mechanism. Therefore, for patients with thin EMT, the perinatal period may require more attention from obstetricians and pediatricians.

Disclosure of interests

All authors have nothing to disclose.

Contribution to authorship

WXL designed the study. DMZ and ZJW were involved in the data extraction and analysis. LMM reviewed the data. DMZ and ZJW was involved in drafting this article. All authors have approved the final version of the manuscript.

Details of ethics approval

This study was approved by the ethics committee of The Third Affiliated Hospital of Zhengzhou University (2020-107).

\section{Funding}

We did not receive any funding for this study.

Acknowledgments

We acknowledge the patients who participated in the study. We also thank American Journal Experts for their professional manuscript-editing service.

\section{References}

1. Vander Borght M, Wyns C. Fertility and infertility: Definition and epidemiology. Clinical biochemistry. 2018 Dec;62:2-10.

2. Calhaz-Jorge C, De Geyter CH, Kupka MS, Wyns C, Mocanu E, Motrenko T, et al. Survey on ART and IUI: legislation, regulation, funding and registries in European countries: The European IVFmonitoring Consortium (EIM) for the European Society of Human Reproduction and Embryology (ESHRE). 2020;2020(1):hoz044.

3. Zhang J, Du M, Li Z, Wang L, Hu J, Zhao B, et al. Fresh versus frozen embryo transfer for full-term singleton birth: a retrospective cohort study. Journal of ovarian research. 2018 Jul 16;11(1):59.

4. Qin JB, Sheng XQ, Wu D, Gao SY, You YP, Yang TB, et al. Worldwide prevalence of adverse pregnancy outcomes among singleton pregnancies after in vitro fertilization/intracytoplasmic sperm injection: a systematic review and meta-analysis. Arch Gynecol Obstet. 2017 Feb;295(2):285-301.

5. Cavoretto P, Candiani M, Giorgione V. Risk of spontaneous preterm birth in singleton pregnancies conceived after IVF/ICSI treatment: meta-analysis of cohort studies. 2018 Jan;51(1):43-53.

6. Pinborg A, Wennerholm UB, Romundstad LB, Loft A, Aittomaki K, Soderstrom-Anttila V, et al. Why do singletons conceived after assisted reproduction technology have adverse perinatal outcome? Systematic review and meta-analysis. Human reproduction update. 2013 Mar-Apr;19(2):87-104.

7. Schieve LA, Meikle SF, Ferre C, Peterson HB, Jeng G, Wilcox LS. Low and very low birth weight in infants conceived with use of assisted reproductive technology. The New England journal of medicine. 2002 Mar 7;346(10):731-7. 
8. Pereira N, Elias RT, Christos PJ, Petrini AC, Hancock K, Lekovich JP, et al. Supraphysiologic estradiol is an independent predictor of low birth weight in full-term singletons born after fresh embryo transfer. Human reproduction. 2017;32(7):1410-7.

9. Zhang J, Du M, Sun L. Supraphysiological estradiol levels on the hCG trigger day are associated with SGA for singletons born from fresh embryo transfer. 2021 May 11:1-8.

10. Zhang J, Sun L, Li Z, Du M. HIGH SERUM ESTRADIOL LEVEL ON THE DAY OF HCG TRIGGER IS ASSOCIATED WITH SMALL FOR GESTATIONAL AGE INFANTS: A RETROSPECTIVE COHORT STUDY. Fertility and sterility. 2018 Sep;110(4):E32-E.

11. Mouhayar Y, Franasiak JM, Sharara FI. Obstetrical complications of thin endometrium in assisted reproductive technologies: a systematic review. Journal of assisted reproduction and genetics. 2019;36(4):607-11.

12. Miwa I, Tamura H, Takasaki A, Yamagata Y, Shimamura K, Sugino N. Pathophysiologic features of "thin" endometrium. Fertility and sterility. 2009 Apr;91(4):998-1004.

13. Guo Z, Xu X, Zhang L, Zhang L, Yan L, Ma J. Endometrial thickness is associated with incidence of small-for-gestational-age infants in fresh in vitro fertilization-intracytoplasmic sperm injection and embryo transfer cycles. Fertility and sterility. 2020;113(4):745-52.

14. Oron G, Hiersch L, Rona S, Prag-Rosenberg R, Sapir O, Tuttnauer-Hamburger M, et al. Endometrial thickness of less than $7.5 \mathrm{~mm}$ is associated with obstetric complications in fresh IVF cycles: a retrospective cohort study. Reproductive biomedicine online. 2018;37(3):341-8.

15. Zhang J, Liu H, Mao X, Chen Q, Si J, Fan Y, et al. Effect of endometrial thickness on birthweight in frozen embryo transfer cycles: an analysis including 6181 singleton newborns. Human reproduction. 2019 Sep 29;34(9):1707-15.

16. Yuan X, Saravelos SH, Wang Q, Xu Y, Li TC, Zhou C. Endometrial thickness as a predictor of pregnancy outcomes in 10787 fresh IVF-ICSI cycles. Reproductive biomedicine online. 2016 Aug;33(2):197-205.

17. Griesinger G, Trevisan S, Cometti B. Endometrial thickness on the day of embryo transfer is a poor predictor of IVF treatment outcome. Human Reproduction Open. 2018;2018(1).

18. Kasius A, Smit JG, Torrance HL, Eijkemans MJ, Mol BW, Opmeer BC, et al. Endometrial thickness and pregnancy rates after IVF: a systematic review and meta-analysis. Human reproduction update. 2014 Jul-Aug;20(4):530-41.

19. Wang X, Du M, Guan Y, Wang B, Zhang J, Liu Z. Comparative neonatal outcomes in singleton births from blastocyst transfers or cleavage-stage embryo transfers: a systematic review and meta-analysis. Reproductive biology and endocrinology : RB\&E. 2017 May 4;15(1):36.

20. Glujovsky D, Farquhar C, Quinteiro Retamar AM, Alvarez Sedo CR, Blake D. Cleavage stage versus blastocyst stage embryo transfer in assisted reproductive technology. The Cochrane database of systematic reviews. 2016 Jun 30(6):Cd002118.

21. Zegers-Hochschild F, Adamson GD, Dyer S, Racowsky C, de Mouzon J, Sokol R, et al. The International Glossary on Infertility and Fertility Care, 2017. Fertility and sterility. 2017 Sep;108(3):393-406.

22. Chung K, Coutifaris C, Chalian R, Lin K, Ratcliffe SJ, Castelbaum AJ, et al. Factors influencing adverse perinatal outcomes in pregnancies achieved through use of in vitro fertilization. Fertility and sterility. 2006 Dec;86(6):1634-41.

23. Rombauts L, Motteram C, Berkowitz E, Fernando S. Risk of placenta praevia is linked to endometrial thickness in a retrospective cohort study of 4537 singleton assisted reproduction technology births. Human reproduction. 2014 Dec;29(12):2787-93. 
24. Marconi N, Raja EA, Bhattacharya S, Maheshwari A. Perinatal outcomes in singleton live births after fresh blastocyst-stage embryo transfer: a retrospective analysis of 67147 IVF/ICSI cycles. Human reproduction. 2019 Sep 29;34(9):1716-25.

25. Dar S, Lazer T, Shah PS, Librach CL. Neonatal outcomes among singleton births after blastocyst versus cleavage stage embryo transfer: a systematic review and meta-analysis. Human reproduction update. 2014 May-Jun;20(3):439-48.

26. Glujovsky D, Blake D, Farquhar C, Bardach A. Cleavage stage versus blastocyst stage embryo transfer in assisted reproductive technology. The Cochrane database of systematic reviews. 2012 Jul 11(7):Cd002118.

27. Casper RF. It's time to pay attention to the endometrium. Fertility and sterility. 2011 Sep;96(3):519-21.

28. Schoots MH, Gordijn SJ, Scherjon SA, Van Goor H, Hillebrands JL. Oxidative stress in placental pathology. Placenta. 2018:S0143400418300705.

29. Ribeiro VC, Santos-Ribeiro S, De Munck N, Drakopoulos P, Polyzos NP, Schutyser V, et al. Should we continue to measure endometrial thickness in modern-day medicine? The effect on live birth rates and birth weight. Reproductive biomedicine online. 2018;36(4):416-26.

30. Gupta S, Malhotra N, Sharma D, Chandra A, Ashok A. Oxidative Stress and its Role in Female Infertility and Assisted Reproduction: Clinical Implications. International Journal of Fertility \& Sterility. 2009;2(4):147-64.

31. Brosens I, Puttemans P, Benagiano G. Placental bed research: I. The placental bed: from spiral arteries remodeling to the great obstetrical syndromes. American journal of obstetrics and gynecology. 2019 Nov;221(5):437-56.

32. Norwitz ER. Defective implantation and placentation: laying the blueprint for pregnancy complications. Reproductive biomedicine online. 2007;14 Spec No 1:101-9.

Table 1 Comparison of demographic and clinical characteristics among the three groups

Item

No. of cases

Maternal age (year)

Body mass index $\left(\mathrm{kg} / \mathrm{m}^{2}\right)$

Duration of infertility (year)

Type of infertility

Primary infertility

Secondary infertility

Infertility diagnosis

Tubal factor

Male factor

Others

Basal FSH(IU/L)

Number of antral follicle count

Methods of ART (\%)

IVF

ICSI

$\mathrm{COH}$ protocols $(\%)$

GnRH-a protocol

GnRH-anti protocol

Duration of ovarian stimulation (d)

Dosage of gonadotropins(IU)

Serum estradiol on the trigger day $(\mathrm{pg} / \mathrm{ml})$
EMT[?]7.5 mm 181

$30(28,35)$

$23.4(20.8,25.0)$

$3.0(1.5,4.0)$

$50.3(91 / 181)$

$49.7(90 / 181)$

$38.1(69 / 181)$

$26.5(48 / 181)$

$35.4(64 / 181)$

$6.6(5.2,7.8)$

$15(10,18)$

$73.5(133 / 181)$

$26.5(48 / 181)$

$70.7(128 / 181)$

$29.3(53 / 181)$

$12(10,14)$

$2550(2025,3562)$

3003.0(1966.5,45 
No. of oocytes retrieved

$10(7,13)$

Note: Data are presented as median (P25,P75) for continuous variable and n (\%) for categorical variable Note: Data are

Table 2 Comparison of neonatal birthweight, gender and malformations among the three groups

Item

No. of cases

Birthweight $(\mathrm{g})$

Gender of newborn (\%)

Male

Female

LBW (\%)

Neonatal malformations (\%)

Note: Data are presented as median(P25,P75) for continuous variable and n (\%) for categorical variable; ${ }^{*}$ shows that there

Table 3 Binary logistic regression analysis to account for confounding variables of low birth weight

Item

Age

Body mass index

Type of infertility (primary/ secondary)

Infertility diagnosis (tubal/male/ others)

Method of ART (IVF/ICSI)

$\mathrm{COH}$ protocols $(\mathrm{GnRH}-\mathrm{a}$ protocol $/ \mathrm{GnRH}$-anti protocol)

The serum estradiol on the trigger day $(<4000 \mathrm{pg} / \mathrm{ml}[?] 4000 \mathrm{pg} / \mathrm{ml})$

$\operatorname{EMT}(\mathrm{mm})$

$7.6^{\sim} 12.0$

$>12.0$

7.5

Gestational weeks

Gender of newborn (male/ female)

Note: Variable entered in the logistics regression model listed. AOR, adjusted odds ratio; CI, confidence interval. EMT, en 